\title{
EXPONENTIAL ATTRACTOR FOR THE THREE- DIMENSIONAL NAVIER-STOKES EQUATION WITH NONLINEAR DAMPING
}

\author{
XUE-LI SONG, FEI LIANG and JUN SU \\ College of Science \\ Xi'an University of Science and Technology \\ Xi'an, 710054 \\ P. R. China \\ e-mail: songxlmath@163.com \\ fliangmath@126.com \\ junsu99@126.com
}

\begin{abstract}
In this paper, we consider the asymptotic behaviour of solutions for three-dimensional incompressible Navier-Stokes equations with nonlinear damping. We show the squeezing property and the existence of exponential attractor for this equation.
\end{abstract}

\section{Introduction}

In this paper, we investigate the following Navier-Stokes equation with nonlinear damping:

2010 Mathematics Subject Classification: 35B40, 35B41.

Keywords and phrases: Navier-Stokes equation, damping, exponential attractor.

Supported by the NSF of China (Tianyuan fund for Mathematics, No. 11426171); and NSF of China (No. 11402194); and Scientific Research Program funded by Shaanxi Provincial Education Department (No. 14JK1474).

Received May 14, 2015

(C) 2015 Scientific Advances Publishers 


$$
\left\{\begin{array}{cc}
u_{t}-\mu \Delta u+(u \cdot \nabla) u+\alpha|u|^{\beta-1} u+\nabla p=f, & (x, t) \in \Omega \times(0, T), \\
\operatorname{div} u=0, & (x, t) \in \Omega \times[0, T), \\
\left.u\right|_{t=0}=u_{0}, & x \in \Omega, \\
u(x, t)=0, & (x, t) \in \partial \Omega \times[0, T),
\end{array}\right.
$$

where $\Omega \subset \mathbb{R}^{3}$ be an open bounded domain with the boundary $\partial \Omega$ smooth enough. $\mu>0$ is the kinematic viscosity of the fluid and $f=f(x)$ is the external body force. The unknown functions here are $u=u(x, t)=\left(u_{1}(x, t), u_{2}(x, t), u_{3}(x, t)\right)$ and $p=p(x, t)$, which stand for the velocity field and the pressure of the flow, respectively. In dampness term, $\beta \geq 1$ and $\alpha>0$ are two constants. The given function $u_{0}=u_{0}(x)$ is the initial velocity.

We will use the following notations in this paper:

$$
\mathcal{V}=\left\{u \in\left(C_{0}^{\infty}(\Omega)\right)^{3}: \operatorname{div} u=0\right\}, \quad H=c l_{\left(L^{2}(\Omega)\right)^{3}} \mathcal{V}, \quad V=c l_{\left(H_{0}^{1}(\Omega)\right)^{3}} \mathcal{V}
$$

where $c l_{X}$ denotes the closure in the space $X$. It is well known that $H, V$ are separable Hilbert spaces and identifying $H$ and its dual $H^{\prime}$, we have $V \hookrightarrow H \hookrightarrow V^{\prime}$ with dense and continuous injections, and $V \hookrightarrow H$ is compact. $H$ and $V$ endowed, respectively, with the inner products

$$
(u, v)=\int_{\Omega} u \cdot v d x, \quad \forall u, v \in H
$$

and

$$
((u, v))=\sum_{i=1}^{3} \int_{\Omega} \nabla u_{i} \cdot \nabla v_{i} d x, \quad \forall u, v \in V
$$

and norms $|\cdot|_{2}=(\cdot, \cdot)^{1 / 2},\|\cdot\|=((\cdot, \cdot))^{1 / 2}$. 
In this paper, $\mathbf{L}^{p}(\Omega)=\left(L^{p}(\Omega)\right)^{3}$, and we use $|\cdot|_{p}$ denotes the norm in $\mathbf{L}^{p}(\Omega)$. The letter $c$ or $c_{i}$ is a generic positive constant, which may change its value from line to line.

We say that $u \in L^{\infty}(0, T ; H) \cap L^{2}(0, T ; V) \cap L^{\beta+1}\left(0, T ; \mathbf{L}^{\beta+1}(\Omega)\right)$ is a weak solution of (1) on $[0, T]$, if

$$
\left\{\begin{array}{c}
\frac{d}{d t}(u, v)+\mu((u, v))+b(u, u, v)+\left(\alpha|u|^{\beta-1} u, v\right)=(f, v), \quad \forall v \in V, \quad \forall t>0, \\
u(0)=u_{0} .
\end{array}\right.
$$

The weak formulation (2) is equivalent to the function equation

$$
\left\{\begin{array}{c}
\frac{d u}{d t}+\mu A u+B(u)+G(u)=f, \quad \forall t>0 \\
u(0)=u_{0}
\end{array}\right.
$$

where $\widetilde{P}$ be the orthogonal projection of $\left(L^{2}(\Omega)\right)^{3}$ onto $H$ and $f \in H$. $A u=-\widetilde{P} \Delta u$ is the Stokes operator defined by $\langle A u, v\rangle=((u, v))$, and $F(u)=\alpha|u|^{\beta-1} u, G(u)=\widetilde{P} F(u) . B: V \times V \rightarrow V^{\prime}$ is a bilinear operator defined by $\langle B(u, v), w\rangle=b(u, v, w) . B(u)=B(u, u)$, where

$$
b(u, v, w)=\sum_{i, j=1}^{3} \int_{\Omega} u_{i} \frac{\partial v_{j}}{\partial x_{i}} w_{j} d x
$$

and $\langle\cdot, \cdot\rangle$ is the duality product between $V$ and $V^{\prime}$.

We call the function pair $(u(x, t), p(x, t))$ the strong solution of problem (1) if it is a weak solution of (1) satisfying

$$
u \in L^{\infty}(0, T ; V) \cap L^{2}\left(0, T ; H^{2}(\Omega)\right) \cap L^{\infty}\left(0, T ; \mathbf{L}^{\beta+1}(\Omega)\right) .
$$

Let us recall some relevant researches in this area. 
In [1], Cai and Jiu investigated the existence and uniqueness of strong solution of system (1) when $\frac{7}{2} \leq \beta \leq 5, u_{0} \in V$. In [2], we showed the strong solutions has global attractors in $V$ and $\mathbf{H}^{2}(\Omega)$ when initial data $u_{0} \in V$. And in [3], we proved the strong solutions has uniform attractors in $V$ and $H^{2}(\Omega)$ when initial data $u_{\tau} \in V$. As far as we know, there are no other results about attractors of system (1).

In this paper, we will discuss the existence of finite dimensional exponential attractor for system (1). In Section 2, we state some basic results on the existence of exponential attractors and recall some known results on the existence and uniqueness of solutions. In Section 3, we establish the Lipschitz continuity of the dynamical system $S(t)$ associated with system (1), and prove the semigroup $S(t)$ satisfies the squeezing property and obtain the existence of the exponential attractor.

\section{Preliminaries}

In this section, we state some basic results on the existence of exponential attractors and recall some known results of strong solutions.

Definition 2.1 ([4]). Assume $\{S(t)\}_{t \geq 0}$ be semigroup defined on $E$. A compact set $M \subset E$ is called an exponential attractor of $\left(\{S(t)\}_{t \geq 0}, E\right)$ if

(1) $S(t) M \subseteq M, \quad \forall t \geq 0$,

(2) $M$ has finite fractal dimension, $d_{F}(M)<+\infty$,

(3) for any bounded set $B$ in $E$, there exist positive constants $c_{0}, c_{1}$ such that

$$
\operatorname{dist}_{V}(S(t) B, M) \leq c_{0} e^{-c_{1} t}, \quad \forall t>0,
$$

where $\operatorname{dist}_{V}(A, B)=\sup _{x \in A} \inf _{y \in B}\|x-y\|_{V}, B$ is a positively invariant set for $S(t)$ in $V$. 
Definition 2.2 ([4]). If for every $\delta \in\left(0, \frac{1}{8}\right)$, there exists a time $t_{*}>0$, an integer $N_{0} \geq 1$, and an orthogonal projection $P_{N_{0}}$ of rank equal to $N_{0}$ such that for every $u$ and $v$ in $B$, either

$$
\left\|S\left(t_{*}\right) u-S\left(t_{*}\right) v\right\| \leq \delta\|u-v\|,
$$

or

$$
\left\|Q_{N_{0}}\left(S\left(t_{*}\right) u-S\left(t_{*}\right) v\right)\right\| \leq\left\|P_{N_{0}}\left(S\left(t_{*}\right) u-S\left(t_{*}\right) v\right)\right\|,
$$

then we call $S(t)$ is squeezing in $B$, where $Q_{N_{0}}=I-P_{N_{0}}$.

Theorem 2.1 ([5]). Suppose (3) satisfies the following conditions:

(1) there exists a compact global attractor $\mathcal{A}$ in $V$,

(2) there exists a compact set B in $V$ which is positively invariant for $S(t)$,

(3) $S(t)$ is squeezing and Lipschitz continuous, that is there exists a bounded function $l(t)$ such that $\|S(t) u-S(t) v\| \leq l(t)\|u-v\|$ for every $u, v \in B$.

Then (3) admits a exponential attractor $M$ for $(S(t), B)$ in $V$ and

$$
M=\bigcup_{0 \leq t \leq t_{*}} S(t) M_{*},
$$

where

$$
M_{*}=\mathcal{A} \bigcup\left(\bigcup_{j=1}^{\infty} \bigcup_{k=1}^{\infty} S\left(t_{*}\right)^{j}\left(E^{(k)}\right)\right)
$$

Moreover,

$$
\begin{gathered}
d_{F}(M) \leq 1+N_{0} \log (1+\sqrt{2} l / \delta) \log \frac{1}{\theta}, \\
\operatorname{dist}_{V}(S(t) B, M) \leq c_{0} e^{-c_{1} t},
\end{gathered}
$$

where $\theta, N_{0}, E^{(k)}$ are defined as in [4], $l$ is the Lipschitz constant for $S\left(t_{*}\right)$ in $B$, and $t_{*}$ is a positive constant. 
In [1], the authors have established the following result:

Theorem 2.2. Suppose that $\beta \geq \frac{7}{2}, u_{0} \in V \cap \mathbf{L}^{\beta+1}(\Omega)$ and $f \in H$. Then there exists a strong solution $(u(x, t), p(x, t))$ to problem (1) satisfying

$$
\begin{gathered}
u \in L^{\infty}(0, T ; V) \cap L^{\infty}\left(0, T ; \mathbf{L}^{\beta+1}(\Omega)\right) \cap L^{2}\left(0, T ; \mathbf{H}^{2}(\Omega)\right), \\
\nabla u|u|^{\frac{\beta-1}{2}} \in L^{2}(0, T ; H), u_{t} \in L^{2}(0, T ; H) .
\end{gathered}
$$

Moreover when $\frac{7}{2} \leq \beta \leq 5$, the strong solution is unique.

Because $\Omega \subset \mathbb{R}^{3}$ is sufficiently regular, so $V \hookrightarrow \mathbf{L}^{\beta+1}(\Omega)\left(\frac{7}{2} \leq \beta \leq 5\right)$, so $V \cap \mathbf{L}^{\beta+1}(\Omega)=V$. In the following, we use $u_{0} \in V$ to replace $u_{0} \in V$ $\cap \mathbf{L}^{\beta+1}(\Omega)$.

In [2], we have established the following results:

Proposition 2.1. Suppose that $\frac{7}{2} \leq \beta \leq 5, u_{0} \in V$ and $f \in H$. Then there exists closed absorbing sets $B_{1}, B_{2}$ in $V, D(A)$, respectively, and

$$
\begin{gathered}
B_{1}=\left\{u \in V:\|u\| \leq \rho_{1}\right\}, \\
B_{2}=\left\{u \in D(A):|A u|_{2} \leq \rho_{2}\right\} .
\end{gathered}
$$

Letting $B=B_{2}$, then $B=B_{2} \subset B_{1} \subset V$, and $S(t) B \subset B$.

Theorem 2.3. Suppose that $\frac{7}{2} \leq \beta \leq 5, u_{0} \in V$ and $f \in H$. Then problem (1) has a global attractor in $V$, which is invariant and compact and attracts every bounded subset of $V$ with respect to the norm topology of $V$. 
Proposition $2.2([6])$.

$$
\begin{gathered}
|b(u, v, w)| \leq\|u\|_{\infty}\|v\||w|_{2}, u \in L^{\infty}, v \in V, w \in H \\
|b(u, v, w)| \leq k\|u\|\|v\|^{1 / 2}|A v|_{2}^{1 / 2}|w|_{2}, u \in V, v \in D(A), w \in H .
\end{gathered}
$$

Now, let us recall the known Gagliardo-Nirenberg inequality as follows.

Lemma 2.1. Let $\Omega=\mathbb{R}^{n}$ or $\Omega \subset \mathbb{R}^{n}$ be a bounded domain with smooth boundary $\partial \Omega$, and $u \in \mathbf{L}^{q}(\Omega), D^{m} u \in \mathbf{L}^{r}(\Omega), 1 \leq q, r \leq \infty$. Then there exists a constant $c$, such that

$$
\left|D^{j} u\right|_{p} \leq c\left|D^{m} u\right|_{r}^{a}|u|_{q}^{1-a}
$$

where

$$
\frac{1}{p}=\frac{j}{n}+a\left(\frac{1}{r}-\frac{m}{n}\right)+(1-a) \frac{1}{q}, 1 \leq p \leq \infty, 0 \leq j \leq m, \frac{j}{m} \leq a \leq 1 ;
$$

$c$ depends only on $(n, m, j, a, q, r)$.

Since the Stokes operator $A$ is positive, self-adjoint operator with a compact inverse, there exists a set of eigenvalues $\lambda_{1}, \lambda_{2}, \lambda_{3}, \ldots$ such that

$$
0<\lambda_{1} \leq \lambda_{2} \leq \lambda_{3} \leq \cdots, \lambda_{N} \rightarrow \infty \text { as } N \rightarrow+\infty,
$$

and corresponding eigenvectors $w_{1}, w_{2}, w_{3}, \ldots$, in $D(A)$, which form a basis of $V$ and orthonormal in $H$, such that

$$
A w_{n}=\lambda_{n} w_{n}, \quad \forall n \in \mathbb{N} .
$$

Let $V_{N}=\operatorname{span}\left\{w_{1}, \ldots, w_{N}\right\}$, then $V_{N}$ is a finite dimensional subspace of $V$. Denote by $P_{N}$ the orthogonal projector from $V$ into $V_{N}$ and we obviously have $\left\|P_{N}\right\| \leq 1$ for each $N \in \mathbb{N}$, and we write $Q_{N}=I-P_{N}$.

In order to establish the existence of the exponential attractor, according to Theorem 2.1, we need only to show the Lipschitz continuity and the squeezing property of the dynamical system $S(t)$ in $B$. That is what we proceed to do in the following section. 


\section{The Exponential Attractor}

In this section, we first establish the Lipschitz continuity of the dynamical system $S(t)$ associated with the Equation (1) in $B$. Then we show the squeezing property for semigroup $S(t)$ and deduce the existence of finite fractal dimensional exponential attractor.

Lemma 3.1. Suppose $\frac{7}{2} \leq \beta \leq 5, f \in H, u_{1}(t), u_{2}(t)$ are two solutions of the problem (1) with initial values $u_{01}, u_{02} \in B$. Then we have

$$
\left\|u_{1}(t)-u_{2}(t)\right\| \leq e^{c_{1} t}\left\|u_{01}-u_{02}\right\|,
$$

where $c_{1}$ is a constant depending only on $\beta$.

Proof. Let $w(t)=u_{1}(t)-u_{2}(t)$. Then by (3) we find that

$$
\begin{gathered}
\frac{d w}{d t}+\mu A w+B\left(u_{1}\right)-B\left(u_{2}\right)+G\left(u_{1}\right)-G\left(u_{2}\right)=0, \\
w(0)=u_{01}-u_{02} .
\end{gathered}
$$

Taking the inner product of (4) with $A w$ in $H$, we infer that

$$
\begin{aligned}
\frac{1}{2} \frac{d}{d t}\|w\|^{2}+\mu|A w|_{2}^{2}= & -b\left(u_{1}, u_{1}, A w\right)+b\left(u_{2}, u_{2}, A w\right)-\left(F\left(u_{1}\right)-F\left(u_{2}\right), A w\right) \\
= & -b\left(u_{1}, w, A w\right)-b\left(w, u_{2}, A w\right)-\left(F\left(u_{1}\right)-F\left(u_{2}\right), A w\right) \\
\leq & c\left\|u_{1}\right\|\|w\|^{1 / 2}|A w|_{2}^{3 / 2}+c\|w\|\left\|u_{2}\right\|^{1 / 2}\left|A u_{2}\right|_{2}^{1 / 2}|A w|_{2} \\
& +\left|F\left(u_{1}\right)-F\left(u_{2}\right)\right|_{2}|A w|_{2} \\
& \leq \frac{3}{4} \mu|A w|_{2}^{2}+c\left(\left\|u_{1}\right\|^{4}+\left\|u_{2}\right\|\left|A u_{2}\right|_{2}\right)\|w\|^{2}+c\left|F\left(u_{1}\right)-F\left(u_{2}\right)\right|_{2}^{2},
\end{aligned}
$$


and because

$$
\begin{aligned}
\left|F\left(u_{1}\right)-F\left(u_{2}\right)\right|_{2}^{2} & =\left.\int_{\Omega}|\alpha| u_{1}\right|^{\beta-1} u_{1}-\left.\alpha\left|u_{2}\right|^{\beta-1} u_{2}\right|^{2} d x \\
& \leq c \int_{\Omega}\left[\left|u_{1}\right|^{\beta-1}|w|+\left.|| u_{1}\right|^{\beta-1}-\left|u_{2}\right|^{\beta-1}|\cdot| u_{2} \mid\right]^{2} d x \\
& \leq c \int_{\Omega}\left|u_{1}\right|^{2(\beta-1)}|w|^{2} d x+c \int_{\Omega}\left[\left|u_{1}\right|^{\beta-2}+\left|u_{2}\right|^{\beta-2}\right]^{2}\left|u_{2}\right|^{2}|w|^{2} d x
\end{aligned}
$$

in the last inequality, we used the fact that

$$
\left|x^{p}-y^{p}\right| \leq c p\left(|x|^{p-1}+|y|^{p-1}\right)|x-y|,
$$

for any $x, y \geq 0$, where $c$ is an absolute constant. So

$$
\begin{aligned}
\left|F\left(u_{1}\right)-F\left(u_{2}\right)\right|_{2}^{2} & \leq c\left|u_{1}\right|_{3(\beta-1)}^{2(\beta-1)}|w|_{6}^{2}+\left.c|| u_{1}\right|^{\beta-2}+\left.\left|u_{2}\right|^{\beta-2}\right|_{6} ^{2}\left|u_{2}\right|_{6}^{2}|w|_{6}^{2} \\
& \leq c|w|^{2}\left[\left|u_{1}\right|_{3(\beta-1)}^{2(\beta-1)}+\left.|| u_{1}|+| u_{2}\right|_{6(\beta-2)} ^{2(\beta-2)}\left\|u_{2}\right\|^{2}\right] \\
& \leq c|| w \|^{2}\left[\left|u_{1}\right|_{3(\beta-1)}^{2(\beta-1)}+\left(\left|u_{1}\right|_{6(\beta-2)}^{2(\beta-2)}+\left|u_{2}\right|_{6(\beta-2)}^{2(\beta-2)}\right)\left\|u_{2}\right\|^{2}\right] .
\end{aligned}
$$

Substituting (7) into (6), and applying Gronwall inequality, we have

$$
\begin{aligned}
\|w(t)\|^{2} \leq & \|w(0)\|^{2} \exp \left\{c \int _ { 0 } ^ { t } \left[\left\|u_{1}\right\|^{4}+\left\|u_{2}\right\|\left|A u_{2}\right|_{2}+\left|u_{1}\right|_{3(\beta-1)}^{2(\beta-1)}\right.\right. \\
& \left.\left.+\left\|u_{2}\right\|^{2}\left(\left|u_{1}\right|_{6(\beta-2)}^{2(\beta-2)}+\left|u_{2}\right|_{6(\beta-2)}^{2(\beta-2)}\right)\right] d s\right\} .
\end{aligned}
$$

Applying Gagliardo-Nirenberg inequality, we obtain

$$
|u|_{3(\beta-1)}^{2(\beta-1)} \leq c|\Delta u|_{2}^{\frac{8(\beta-2)}{\beta+7}}|u|_{\beta+1}^{\frac{2(\beta+1)^{2}}{\beta+7}},|u|_{6(\beta-2)}^{2(\beta-2)} \leq c|\Delta u|_{2}^{\frac{2(5 \beta-13)}{\beta+7}}|u|_{\beta+1}^{\frac{2\left(\beta^{2}-1\right)}{\beta+7}} .
$$

Because $V \hookrightarrow L^{\beta+1}(\Omega)$, so there exists a positive constant $\rho_{3}$, such that $|u|_{\beta+1} \leq \rho_{3}$ when $u \in B$. 
Because $u_{1}, u_{2} \in B$, and thanks to (9), there must exists a positive constant $c_{1}$, such that

$$
\|w(t)\|^{2} \leq\|w(0)\|^{2} e^{2 c_{1} t}=e^{2 c_{1} t}\left\|u_{01}-u_{02}\right\|^{2} .
$$

Lemma 3.2. Assume that $u_{1}(t)$ and $u_{2}(t)$ are two solutions of problem (1) with initial values $u_{01}, u_{02} \in B$. Then $q=Q_{N}\left(u_{1}-u_{2}\right)$ satisfies that

$$
\|q(t)\|^{2} \leq\left(e^{-\mu \lambda \lambda_{N+1} t}+\frac{c_{2}}{2 c_{1}+\mu \lambda_{N+1}} e^{2 c_{1} t}\right)\|w(0)\|^{2} .
$$

Proof. Let $w(t)=u_{1}(t)-u_{2}(t), q(t)=Q_{N} w(t)$. Applying $Q_{N}$ to

$$
\frac{d w}{d t}+\mu A w=-B\left(u_{1}\right)+B\left(u_{2}\right)-\widetilde{P} F\left(u_{1}\right)+\widetilde{P} F\left(u_{2}\right),
$$

we obtain

$$
\frac{\partial q(t)}{\partial t}+\mu A q=Q_{N}\left(B\left(u_{2}\right)-B\left(u_{1}\right)\right)+Q_{N}\left(\widetilde{P} F\left(u_{2}\right)-\widetilde{P} F\left(u_{1}\right)\right) .
$$

Taking the inner product of (10) with $A q$ in $H$, we find that

$\frac{1}{2} \frac{d}{d t}\|q\|^{2}+\mu|A q|_{2}^{2}=\int_{\Omega} Q_{N}\left(B\left(u_{2}\right)-B\left(u_{1}\right)\right) A q d x+\int_{\Omega} Q_{N}\left(\widetilde{P} F\left(u_{2}\right)-\widetilde{P} F\left(u_{1}\right)\right) A q d x$.

Because

$$
\begin{aligned}
\left|\int_{\Omega} Q_{N}\left(\widetilde{P} F\left(u_{2}\right)-\widetilde{P} F\left(u_{1}\right)\right) A q d x\right| & \leq\left|F\left(u_{1}\right)-F\left(u_{2}\right)\right|_{2} \cdot|A q|_{2} \\
& \leq \frac{1}{\mu}\left|F\left(u_{1}\right)-F\left(u_{2}\right)\right|_{2}^{2}+\frac{\mu}{4}|A q|_{2}^{2},
\end{aligned}
$$

and

$$
\begin{aligned}
\left|\int_{\Omega} Q_{N}\left(B\left(u_{2}\right)-B\left(u_{1}\right)\right) A q d x\right| & \leq\left|B\left(u_{2}\right)-B\left(u_{1}\right)\right|_{2} \cdot|A q|_{2} \\
& \leq \frac{1}{\mu}\left|B\left(u_{2}\right)-B\left(u_{1}\right)\right|_{2}^{2}+\frac{\mu}{4}|A q|_{2}^{2},
\end{aligned}
$$




$$
\begin{aligned}
\left|B\left(u_{2}\right)-B\left(u_{1}\right)\right|_{2}^{2} & =\int_{\Omega}\left[B\left(u_{2}, u_{2}-u_{1}\right)+B\left(u_{2}-u_{1}, u_{1}\right)\right]^{2} d x \\
& \leq 2\left(\left|B\left(u_{2}, u_{2}-u_{1}\right)\right|_{2}^{2}+\left|B\left(u_{2}-u_{1}, u_{1}\right)\right|_{2}^{2}\right) \\
& \leq 2\left\|u_{2}\right\|_{\infty}^{2}\|w\|^{2}+c\left\|u_{1}\right\|\left|A u_{1}\right|_{2}\|w\|^{2},
\end{aligned}
$$

and since $\lambda_{N+1}\|q\|^{2} \leq|A q|_{2}^{2}$, combining (12)-(14) with (11), we have

$$
\begin{aligned}
\frac{d}{d t}\|q\|^{2}+\mu \lambda_{N+1}\|q\|^{2} \leq & c\left\{\left|u_{1}\right|_{3(\beta-1)}^{2(\beta-1)}+\left[\left|u_{1}\right|_{6(\beta-2)}^{2(\beta-2)}+\left|u_{2}\right|_{6(\beta-2)}^{2(\beta-2)}\right]\left\|u_{2}\right\|^{2}\right. \\
& \left.+\left\|u_{2}\right\|_{\infty}^{2}+\left\|u_{1}\right\|\left|A u_{1}\right|_{2}\right\}\|w\|^{2} .
\end{aligned}
$$

Because $u_{2} \in B$, so $\left|A u_{2}\right|_{2} \leq \rho_{2}$. Applying Agmon's inequality, there exists a positive constant $\rho_{4}$ such that

$$
\left\|u_{2}\right\|_{\infty} \leq \rho_{4}
$$

So when $u_{01}, u_{02} \in B$,

$$
\frac{d}{d t}\|q\|^{2}+\mu \lambda_{N+1}\|q\|^{2} \leq c_{2}\|w\|^{2} \leq c_{2} e^{2 c_{1} t}\|w(0)\|^{2}
$$

for some positive constant $c_{2}$.

Then it follows from Gronwall lemma that

$$
\begin{aligned}
\|q\|^{2} & \leq\|q(0)\|^{2} e^{-\mu \lambda_{N+1} t}+\frac{c_{2}}{2 c_{1}+\mu \lambda_{N+1}} e^{2 c_{1} t}\|w(0)\|^{2} \\
& \leq\left(e^{-\mu \lambda_{N+1} t}+\frac{c_{2}}{2 c_{1}+\mu \lambda_{N+1}} e^{2 c_{1} t}\right)\|w(0)\|^{2}
\end{aligned}
$$

which concludes the proof of Lemma 3.2.

We now show the squeezing property.

Lemma 3.3. Suppose $\frac{7}{2} \leq \beta \leq 5$ and $f \in H$, then the semigroup $S(t)$ associated with problem (1) is squeezing in B. 
Proof. Let $t_{*}>0$ fixed, and $w(t)=u_{1}(t)-u_{2}(t)$. We now assume that

$$
\left\|P_{N} w\left(t_{*}\right)\right\| \leq\left\|Q_{N} w\left(t_{*}\right)\right\|
$$

Then we can deduce

$$
\begin{aligned}
\left\|w\left(t_{*}\right)\right\|^{2} & =\left\|P_{N} w\left(t_{*}\right)\right\|^{2}+\left\|Q_{N} w\left(t_{*}\right)\right\|^{2} \\
& \leq 2\left\|Q_{N} w\left(t_{*}\right)\right\|^{2} \\
& \leq 2\left(e^{-\mu \lambda_{N+1} t_{*}}+\frac{c_{2}}{2 c_{1}+\mu \lambda_{N+1}} e^{2 c_{1} t_{*}}\right)\|w(0)\|^{2} \\
& \leq 2\left(e^{-\mu \lambda_{1} t_{*}}+\frac{c_{2}}{2 c_{1}+\mu \lambda_{N+1}} e^{2 c_{1} t_{*}}\right)\|w(0)\|^{2} .
\end{aligned}
$$

Let $t_{*}$ be large enough so that

$$
e^{-\mu \lambda_{1} t_{*}} \leq \frac{1}{256}
$$

Next we choose $N_{0}$ large enough so that

$$
\frac{c_{2}}{2 c_{1}+\mu \lambda_{N+1}} e^{2 c_{1} t_{*}}<\frac{c_{2}}{\mu \lambda \lambda_{N+1}} e^{2 c_{1} t_{*}} \leq \frac{1}{256}
$$

From (18)-(20), we obtain that

$$
\left\|w\left(t_{*}\right)\right\|^{2} \leq \frac{1}{64}\|w(0)\|^{2}
$$

(21) completes the proof of Lemma 3.3.

Theorem 3.1. Suppose $\frac{7}{2} \leq \beta \leq 5$ and $f \in H$, then there exists $N_{0}$ large enough such that

$$
\lambda_{N_{0}+1} \geq \frac{256 c_{2}}{\mu} 2^{16 c_{1} /\left(\mu \lambda_{1}\right)}
$$


For the semigroup $S(t),\left(\{S(t)\}_{t \geq 0}, B\right)$ admits an exponential attractor $M$, and

$$
d_{F}(M) \leq 1+16 c_{3} \sqrt{c_{2} / \mu} 2^{8 c_{1} /\left(\mu \lambda_{1}\right)}
$$

Proof. From Lemmas 3.1 and 3.3, we have known that $\{S(t)\}_{t \geq 0}$ is Lipschitz continuous and squeezing in $B$, by Theorem 2.1, we know $\left(\{S(t)\}_{t \geq 0}, B\right)$ admits an exponential attractor $M$. We note that, $\lambda_{N} \sim N^{2}$, and let $\delta, \theta$ be fixed, thus obtain the estimate (22) of fractal dimension. Theorem 3.1 therefore is proved.

\section{Acknowledgements}

The authors will thank the anonymous referee for their valuable suggestions and comments.

\section{References}

[1] X. Cai and Q. Jiu, Weak and strong solutions for the incompressible Navier-Stokes equations with damping, J. Math. Anal. Appl. 343 (2008), 799-809.

[2] X. Song and Y. Hou, Attractors for the three-dimensional incompressible NavierStokes equations with damping, Discrete and Continuous Dynamical Systems 31 (2011), 239-252.

[3] X. Song and Y. Hou, Uniform attractors for three-dimensional Navier-Stokes equations with nonlinear damping, Journal of Mathematical Analysis and Applications 422 (2015), 337-351.

[4] A. Eden, C. Foias, B. Nicolaenko and R. Temam, Exponential Attractors for Dissipative Evolution Equations, Paris and Wiley, New York, Masson, Research in Applied Mathematics, 1994.

[5] Z. Dai and B. Guo, Inertial fractal sets for dissipative Zakharov system, Acta Math. Appl. Sinica 13 (1997), 279-288.

[6] James C. Robinson, Infinite-Dimensional Dynamical Systems, an Introduction to Dissipative Parabolic PDEs and the Theory of Global Attractors, Cambridge University Press, 2001. 